\title{
Development of a new "6-axis" force connected sensor.
}

\author{
Romain LEFORT ${ }^{1}$, Arnaud DECATOIRE ${ }^{2}$, Malek ABDI ${ }^{3}$, Patrick LACOUTURE ${ }^{4}$, and \\ Raymond BUISSON ${ }^{5}$ \\ ${ }^{1}$ CRITT-Sport \& Loisirs, 21, rue Albert Einstein Z.A. du Sanital, 86100 Châtellerault, France \\ ${ }^{2}$ Université de Poitiers, Institut P' UPR 3346 CNRS SP2MI - Téléport 2, France \\ ${ }^{3}$ WTechnologies, 10, avenue John Fitzgerald Kennedy, 63500 Issoire, France \\ ${ }^{4}$ Institut Pprime, UPR 3346 CNRS, Université de Poitiers, ENSMA, France \\ ${ }^{5}$ RBConsultant, 16, rue de la carrière, 60300 Senlis,France
}

\begin{abstract}
This new sensor project has been initiated mainly in order to take measurements in the field of biomechanics during motions of human bodies. For that, it's necessary to detect the efforts at the contacts with these human bodies in real situation, such as during working, walking, running, biking and so on.
\end{abstract}

Up to now, most of 6 components force sensors which are used, for instance are sensors with each component measuring device as perfectly as possible decoupled from each other's. This leads to expansive or very expansive sophisticated sensors.

The present sensor is a stand-alone wireless, small sized 6-axis force sensor with a powerful and precise conditioning and acquisition system. The sensitive cell is a raw Stewart mechanical structure (strain-gages based) with, conversely to usual multicomponent sensors, force and moment components not decoupled at all, but optimally coupled.

Owing to the powerful numerical capabilities of the sensor, the 6 effective components of a given mechanical action are instantaneously computed. Thanks to that, even for small quantity production, the sensor cost price is significantly reduced. This reduction is bigger for larger quantity productions like for: robotics, machine tools, hoisting machines...

Added to the sensor design, the project include also a theoretical mechanical research in order to find an accurate calibration method, as easy as possible to be performed. This results in calibration tests needing only a standard traction-compression test machine running with mechanical effects decoupling tools dimensioned so that the calibration relative uncertainty is kept below $1 \%$. With that, only 6 elementary loading tests have to be applied to the sensor. The whole sequence of calibration is done automatically, completely governed by a powerful calculation and acquisition software.

All the raw tests results (strain in $\mu \mathrm{m} / \mathrm{m}$ ) are automatically collected, converted and analyzed. At the end of the numerical treatment of each set of measurements, all the calibration data attesting the traceability to the International System of units (SI) of the sensor, including :

- raw calibration results,

- $\quad$ sensitivities coefficients matrix needed for later data reduction and conversion in solicitation components (force and moment), 
- $\quad$ sensor performances characteristic curves (non-linearity, hysteresis error curve, zero shift error, etc.),

- calibrations uncertainties,

are stored in the computer memory.

The calibration matrix is then uploaded on the sensor. So, the measurement results (values of solicitations components) are directly expressed in mechanical units traceable to SI.

This sensor is able to perform high data rate wireless streaming with time-synchronization protocol or low data rate transmissions compatible with IOT connectivity.

The following paper describes and comments most important engineering job sequences and calibration results.

It's also an example of future connected sensors structures able to gather, not only the staff needed to give accurate high levels measurement results, but also all the key pieces of information's relative to the measurement traceability proof and quality management, all of them being instantaneously available on the net (IOT).

This research and development job got the funding of FEDER-FSE-2014-2020 Nouvelle-Aquitaine program and of CRITT-Sport et Loisirs.

\section{Introduction}

Experimentation and research in biomechanics are more and more based on a lot of accurate measured data. The relevant parameters in this field are the components of mechanical solicitations. Consequently, it has been decided to build a new type of sensors able to measure at the same time all the components of such mechanical solicitations; that is to say a 6-axis (or 6 components) force sensors.

The following article deals with all the challenges of such a project:

- Design of a cheap sensor with not perfectly uncoupled components measuring devices,

- Description of the gaging of the sensitive element and it's onboard electronics and micro-informatics conditioning system directly embedded inside the sensor,

- Definition of a special calibration method and engineering and building of calibration tools to be used on a conventional testing machine,

- Description of the analysis technics and the use of the calibration data,

- Characterization of the sensor metrological performances,

- Uses examples in the field of biomechanics and further projects of uses in industrial field.

\section{Mechanical specifications of the sensor to be engineered}

In order to get sound measured data in biomechanical area especially those dedicated to motion analysis, it's necessary to measure mechanical solicitations (efforts, torques and moments) at human/ environment junctions. In mechanical theory, it's the use to consider 
that the whole mechanical actions applied to a solid body constitute a force twister. At a given particular point of this solid body, this force twister is defined by 2 vectors:

An effort resultant vector $\rightarrow\left|\begin{array}{l}F_{x} \\ F_{y} \\ F_{z}\end{array}\right|$ and a moment resultant vector $\rightarrow\left|\begin{array}{c}M_{x} \\ M_{y} \\ M_{z}\end{array}\right|$.

Each one of these vectors can be expressed by three components in the same three dimensional orthogonal axis system.

Measurand definition (physical quantity to be measured by the sensor) includes the following items:

- $\quad$ the physical quantity to be measured is a 6 components mechanical solicitation (3 effort components and 3 moment components),

- measuring intervals are $\pm 8000 \mathrm{~N}$ for the effort components and $\pm 160 \mathrm{Nm}$ for the moment components,

- the working temperature range is: $-20^{\circ} \mathrm{C}$ up to $+50^{\circ} \mathrm{C}$,

- the working moisture range rate is: $40 \%$ to $95 \%$,

- chocks and vibrations that the sensor must accept are: $5 \mathrm{~g}$ half-sine from 1 to 100 $\mathrm{ms}$ for chocks and $3 \mathrm{~g}$ sinusoidal from 0.1 to $25 \mathrm{~Hz}$ for the vibrations,

- the maximal permissible measurement error is $1 \%$ of the measuring range,

- the band-pass of the sensor goes from continuous to $500 \mathrm{~Hz}$; the rising time is: 1 ms.

\section{Engineering of the sensor}

\subsection{Design and running principles of a 6 components effort sensor}

Frequently, 6 components effort sensors called 6 components weighing machine have one independent measuring device per component. These 6 devices, generally are perfectly uncoupled one from the others. This leads to sensors with very complex structures, difficult to fabricate and consequently expensive.

On the contrary, in the present sensor, primary measuring signals it gives are intentionally deeply correlated but in a discriminant way, thanking to a design allying 3 building and functional concepts. Firstly, there is the choice of a sensitive element having the shape of a Stewart platform. Secondly, the installation of an optimized gaging method with resistive foil strain gages and thirdly, the integration full inside the sensor of all the electronics needed to make the sensor self-running and with aboard intelligence. So, with this whole set, it is possible to get simultaneously and immediately all the components of the mechanical solicitation applied on the sensor.

\subsection{Description of the Stewart platform shape sensor sensible element, it's gaging and it's dimensioning}


As the sensible element is a metallic part working in it's perfectly elastic domain, the dependence laws between strain gages signals and pieces of information Fx, Fy, Fz, Mx, My and Mz are linear laws. To get these pieces of information, it's necessary to have, at least, 6 linear relationships independent one another. This is got by a judicious choice of the sensible element (see figure 1 up right) and by a good quantity and a right positioning of strains gages put together to form 6 Wheatstone bridges. This sensible element is small shaped: $53 \mathrm{~mm}$ in diameter and $27 \mathrm{~mm}$ in height. It consists in 6 beams in tractioncompression, gaged with strain gages. When the effort to be measured changes of type (for instance, change from an effort Fx to an effort Fy), the relatives values arrangement of the signals given by the strain gages are completely modified.

Shape and sizes of all of the mechanical parts of the sensors are defined during a deep analysis where all the main mechanical phenomenon's which arise during the use of the sensor are modelled. These mathematical models has been used to optimize all the critical elements of the sensor structure (sensible element beams, bolting junctions for the securing of the sensor, effect of the stresses concentrations in some restricted spots where they appear) so that the signals given by strain gages Wheatstone bridges be as better proportional to the mechanical solicitations to be measured as possible, with as low as possible hysteresis and zero unreturned. They also are used to be sure of the mechanical resistance and in order to eliminate residual strains and/or cracks and/or failure in use.

\subsection{Aboard electronic for strain gages signals analysis}

Strain gages are passive measuring devices. In order to be used, they need an electrical power source and an electronic conditioning of the analog signals they give.

Consequently, the following devices are included inside the sensor casing on a printed circuit board:

- a regulated and stabilized electrical power supply for strain gages,

- an analogic-numeric converter ADS131E08 (24 bits, 64 kSPS, 8 simultaneous channels) converting in numerical form the analog signals given by the strain gages,

- a microcontroller nRF52832 lying on ARM Cortex M4F structure, with $512 \mathrm{kB}$ of flash memory with a wireless communication at $2 \mathrm{Mbps}$.

This onboard electronic is powered by a LiPo (3-7V $150 \mathrm{mAh})$ battery. 

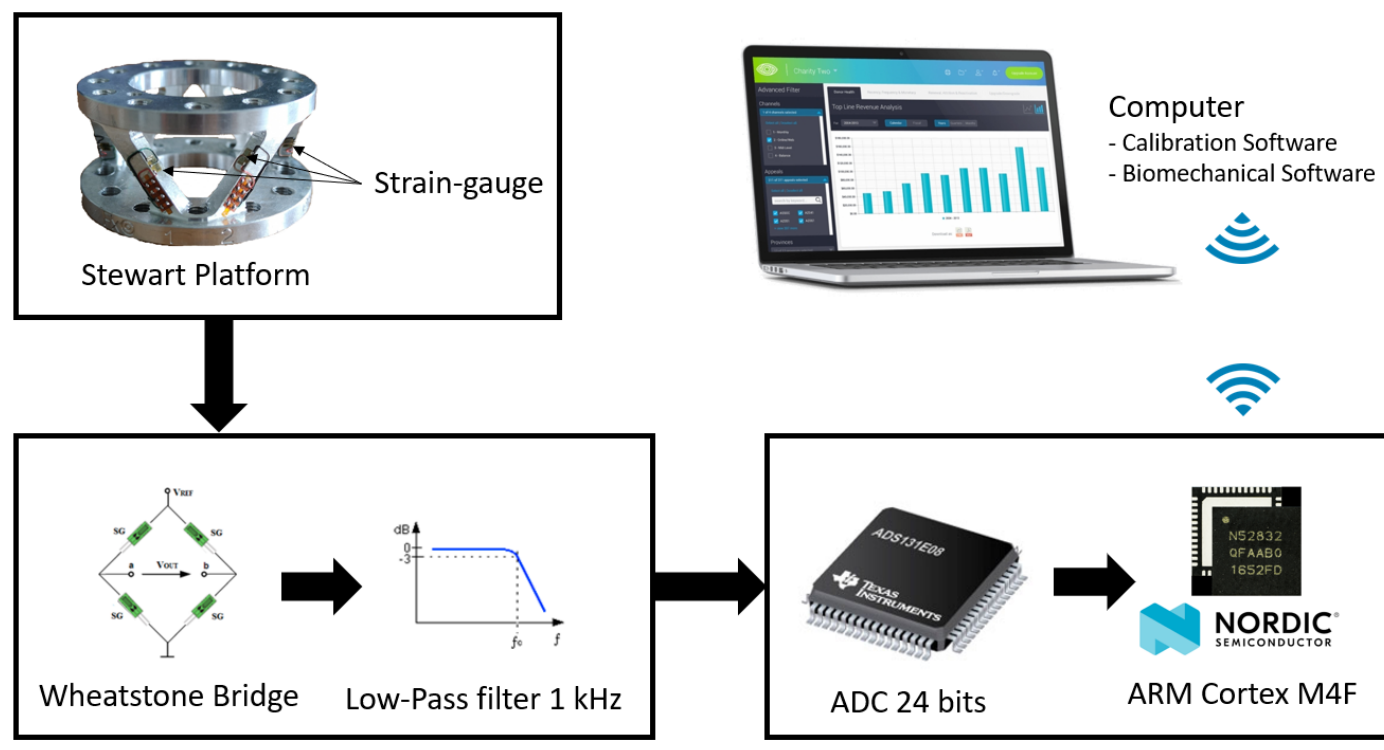

Conditionning circuit (a)

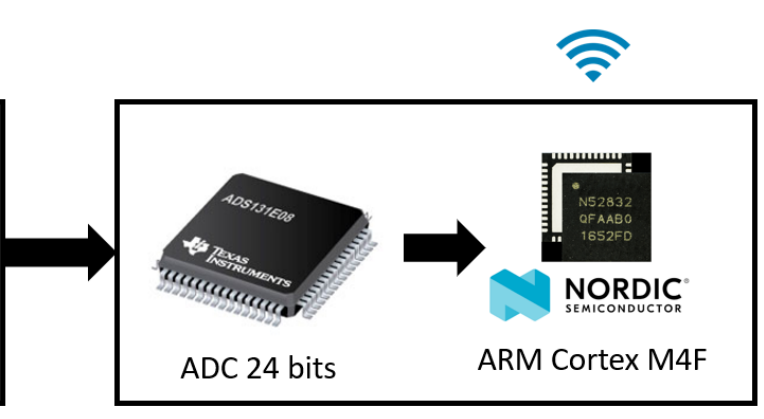

Data acquisition system (b)

Fig. 1: System block diagram to explain the transmission path between the sensor and the computer

Figure 1 depicts this electronic. This one converts in numerical form the analog signals given by the strain gages at reception of the adequate user command (a) in order to save them inside an informatics file. The frequency of this acquisition is defined by the user, previously. These digital signals are then converted in mechanical solicitations components, saved in a buffer then sent, by radio emission, to a reception station where they are saved a new time, treated and displayed as pieces of data (b).

In calibration case, numerical treatments of strain gages signals consists in calculating inverse sensitive coefficients matrix. This one is used to convert resistive strain gages signals into the 6 mechanical solicitation components (6 twister components). It is downloaded inside the flash memory of the microcontroller at the end of the calibration process. These figures are explained in details ahead in $\S 3$ and 4.

\section{Method for calibration and strain gages data use}

The first goal of the calibration tests is to get relationships linking twister components to strain gages Wheatstone bridges signals $\left(\varepsilon_{\mathrm{ti}}\right)$ located on sensor sensible element. Whatever is mechanical solicitation type applied to the sensor, all beams Wheatstone bridges will roughly always give non-null signals (even with only one mechanical solicitation component: Fx for instance, strain gages Wheatstone bridges of the 6 beams will give nonnull signals). The magnitudes of all these signals are proportional to the mechanical solicitation to be measured (Fx in this case).

For instance, in the case of the application of the only one Fx component, each beam is characterized by a sensitive coefficient specific to the measurement with this mechanical solicitation component applied alone. These coefficients may be designed by: $b_{1 F x}$ or $b_{11}$ for beam $n^{\circ} 1, b_{2 \mathrm{Fx}}$ or $\mathrm{b}_{21}$ for beam $\mathrm{n}^{\circ} 2$, etc. and $\mathrm{b}_{6 \mathrm{Fx}}$ or $\mathrm{b}_{61}$ for beam $\mathrm{n}^{\circ} 6$. 
It's the same thing for each of the 5 other elementary mechanical solicitation components (Fy, Fz, Mx, My and Mz). For each of them, there is also 6 sensitivity coefficients $\left(\mathrm{b}_{1 \mathrm{Fy}}\right.$ or $b_{12}$ for beam $n^{\circ} 1$, etc. up to $b_{6 \mathrm{Mz}}$ or $b_{66}$ for beam $n^{\circ} 6$.

So, if the mechanical solicitation applied on the sensor has 6 components: Fx, Fy, Fz, Mx, My and Mz all of them not null, then, as it is assumed that the mechanical behavior of the sensible element of the sensor is linear, the strain gages Wheatstone bridge signal of each beam of the sensitive element is the algebraic sum of the signals given by this same strain gages Wheatstone bridge under the action of each of the 6 elementary mechanical solicitation component.

This is mathematically represented by the following matrix product:

$$
\left|\begin{array}{c}
\varepsilon_{\mathrm{t} 1} \\
\varepsilon_{\mathrm{t} 2} \\
\varepsilon_{\mathrm{t} 3} \\
\varepsilon_{\mathrm{t} 4} \\
\varepsilon_{\mathrm{t} 5} \\
\varepsilon_{\mathrm{t} 6}
\end{array}\right|=\left|\begin{array}{llllll}
\mathrm{b}_{11} & \mathrm{~b}_{12} & \mathrm{~b}_{13} & \mathrm{~b}_{14} & \mathrm{~b}_{15} & \mathrm{~b}_{16} \\
\mathrm{~b}_{21} & \mathrm{~b}_{22} & \mathrm{~b}_{23} & \mathrm{~b}_{24} & \mathrm{~b}_{25} & \mathrm{~b}_{26} \\
\mathrm{~b}_{31} & \mathrm{~b}_{32} & \mathrm{~b}_{33} & \mathrm{~b}_{34} & \mathrm{~b}_{35} & \mathrm{~b}_{36} \\
\mathrm{~b}_{41} & \mathrm{~b}_{42} & \mathrm{~b}_{43} & \mathrm{~b}_{44} & \mathrm{~b}_{45} & \mathrm{~b}_{46} \\
\mathrm{~b}_{51} & \mathrm{~b}_{52} & \mathrm{~b}_{53} & \mathrm{~b}_{54} & \mathrm{~b}_{55} & \mathrm{~b}_{66} \\
b_{61} & b_{62} & b_{63} & b_{64} & b_{65} & b_{66}
\end{array}\right| \cdot\left|\begin{array}{c}
\mathrm{F}_{\mathrm{x}} \\
\mathrm{F}_{\mathrm{y}} \\
\mathrm{F}_{\mathrm{z}} \\
\mathrm{M}_{\mathrm{x}} \\
\mathrm{M}_{\mathrm{y}} \\
\mathrm{M}_{\mathrm{z}}
\end{array}\right|
$$

All the components of $6 \times 6$ matrix (mechanical solicitation sensitivity coefficient matrix) have to be defined from calibration tests results. Each test with an elementary mechanical solicitation component will give 6 sensitivity coefficients. In order to have the whole 36 coefficients of the sensitivity coefficient matrix, 6 calibration tests as discriminant one to each other as possible have to be done. The optimum tests organization consists in doing 6 tests each of them being as close as possible to the 6 tests with one of the 6 elementary mechanical solicitation component.

For that, the sensor is loaded with a tension-compression test machine (Instron $336950 \mathrm{kN}$ with a $1 \%$ error for efforts between $100 \mathrm{~N}$ and $250 \mathrm{~N}$ and a $0.5 \%$ error for efforts between $250 \mathrm{~N}$ and $50 \mathrm{kN}$ ) with a calibrated load cell and mechanical solicitation application adapters generating the 6 mechanical solicitation components (6 axis) (see figure 2). 


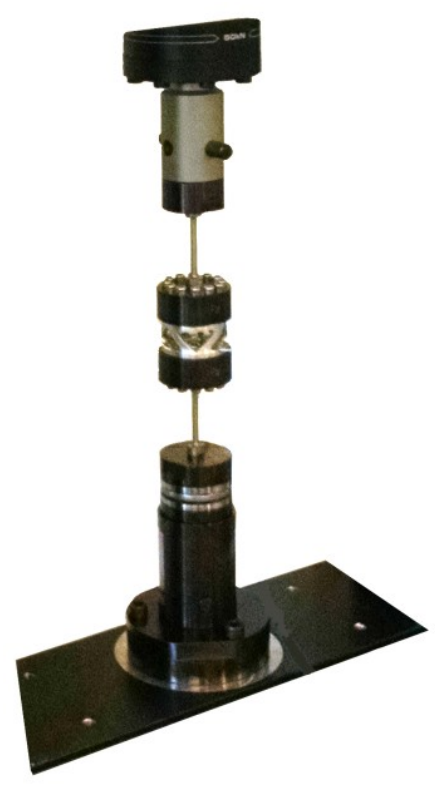

Fig. 2: Picture of the sensor installed on the tension-compression test machine by the mean of a mechanical solicitation application adapter (Fz component adapter in this case).

In real way, each test gives 6 linear equations with, in theory, 36 unknown coefficients. 6 calibration tests, independent one from the others, will give a 36 linear equations system with 36 unknown coefficients. So, in theory, there is no problem to calculate the 36 sensitivity coefficients, except that the resolution of a 36 linear equations system is a tedious job. Hopefully, most of the equations are incomplete equations and the resolution of the equations system is largely simplified.

But, in order to use the sensor, this is not the $b_{i j}$ coefficients which have to be used, but relationships which, on the contrary, give the values of mechanical solicitations components in starting from the strain gages Wheatstone bridges signals. As the mechanical behavior of the sensible element of the sensor is linear, these relationships are also linear relationships and their coefficients comes from the previous coefficients by means a $6 \times 6$ matrix inversion operation. The result is a new $6 \times 6$ matrix called "invers sensitivity coefficients matrix": $\left|\begin{array}{llllll}a_{11} & a_{12} & a_{13} & a_{14} & a_{15} & a_{16} \\ a_{21} & a_{22} & a_{23} & a_{24} & a_{25} & a_{26} \\ a_{31} & a_{32} & a_{33} & a_{34} & a_{35} & a_{36} \\ a_{41} & a_{42} & a_{43} & a_{44} & a_{45} & a_{46} \\ a_{51} & a_{52} & a_{53} & a_{54} & a_{55} & a_{56} \\ a_{61} & a_{62} & a_{63} & a_{64} & a_{65} & a_{66}\end{array}\right|$.

The inversion of a $6 \times 6$ matrix is a large calculation which is similar to the resolution of 36 linear equations system (Kramer system) with zero divides risk. But, the matrix to be inverted is not any matrix. It's a matrix coming from calibration tests, so that it's possible 
to arrange the equations order in order to avoid zero divide risk. Owing to that, the matrix inversion calculations become easier and faster.

With this new matrix, when the sensor is used, the measured signals: $\varepsilon_{t 1}, \varepsilon_{t 2}, \varepsilon_{t 3}, \varepsilon_{t 4}, \varepsilon_{t 5}$ and $\varepsilon_{t 6}$

which are got are converted in effort mechanical solicitations components in using the following matrix relationship: $\left|\begin{array}{c}F_{x} \\ F_{y} \\ F_{z} \\ M_{x} \\ M_{y} \\ M_{z}\end{array}\right|=\left|\begin{array}{llllll}a_{11} & a_{12} & a_{13} & a_{14} & a_{15} & a_{16} \\ a_{21} & a_{22} & a_{23} & a_{24} & a_{25} & a_{26} \\ a_{31} & a_{32} & a_{33} & a_{34} & a_{35} & a_{36} \\ a_{41} & a_{42} & a_{43} & a_{44} & a_{45} & a_{46} \\ a_{51} & a_{52} & a_{53} & a_{54} & a_{55} & a_{56} \\ a_{61} & a_{62} & a_{63} & a_{64} & a_{65} & a_{66}\end{array}\right| \cdot\left|\begin{array}{c}\varepsilon_{t 1} \\ \varepsilon_{t 2} \\ \varepsilon_{t 3} \\ \varepsilon_{t 4} \\ \varepsilon_{t 5} \\ \varepsilon_{t 6}\end{array}\right|$.

\section{Calibration tests realization and got results}

\subsection{Calibration tests principle and particularities in the case of a 6 axis effort sensors}

The sensor calibration tests are done accordingly to their definition given in the international metrological document entitled: « International vocabulary of metrology Basic and general concepts and associated terms" (VIM).

It consists in a comparison of the sensor signals with reference signals having a wellcontrolled trueness and in using a comparison process well-controlled also.

The trueness well-control of reference signals and of the process are got by the uncertainties determination on all measured quantities and in doing all what is needed in order to keep them below a given limit.

All of that is the mandatory needs in order to assume the traceability to the International System of units (SI) of the further measurements results given by the sensor.

The main particularity of non-coupled 6 components effort sensor ( 6 axis effort sensor) is that the comparisons cannot be done immediately on the measurements signals got during the calibration tests.

These signals must firstly be used to calculate the 36 components of the inverse sensitivity coefficients matrix.

So, in order to satisfy the further well-control of all uncertainties, everything is done in order that all the errors that may arise later all along the calibration process are previously identified and special disposition are taken in order that their magnitudes are under control and maintained below an acceptable limit.

In order to fulfill all the requirements of international metrology, the calibration tests of a 6 component effort sensor which give sensitivity coefficients matrix and inverse sensitivity coefficients matrix must be followed either by a repetition of the calibration tests with the mechanical solicitations components computation inside the sensor turned on or by a re 
treatment of the previous calibration tests results with the inverse sensitivity coefficient matrix in order to compute the values of the mechanical solicitations components signals the sensor should had given during these previous calibration tests. Then, in the first possibility as well as in the second, the sensor signals have to be compared to the reference signals.

\subsection{Design and size definition of calibration mechanical solicitations application adapters}

Each of the 6 calibration tests consists in loading the sensor either with an elementary mechanical solicitation or with a mechanical solicitation as close as possible to one of the 6 elementary mechanical solicitation. Tension-compression test machine gives only a tension or a compression effort in the direction of its loading axis. Mechanical solicitation application adapters are mechanical devices which transform this effort into shearing forces or in moment to be applied to the sensor. Figure 2 shows the mechanical solicitation application adapter with apply the elementary effort Fz on the sensor. On figure 3, there are mechanical solicitation application adapters which apply, for instance, elementary mechanical solicitation Fx and mechanical solicitation Mz. Same mechanical solicitations application adapters are for elementary mechanical solicitations Fy and moments Mx and My.
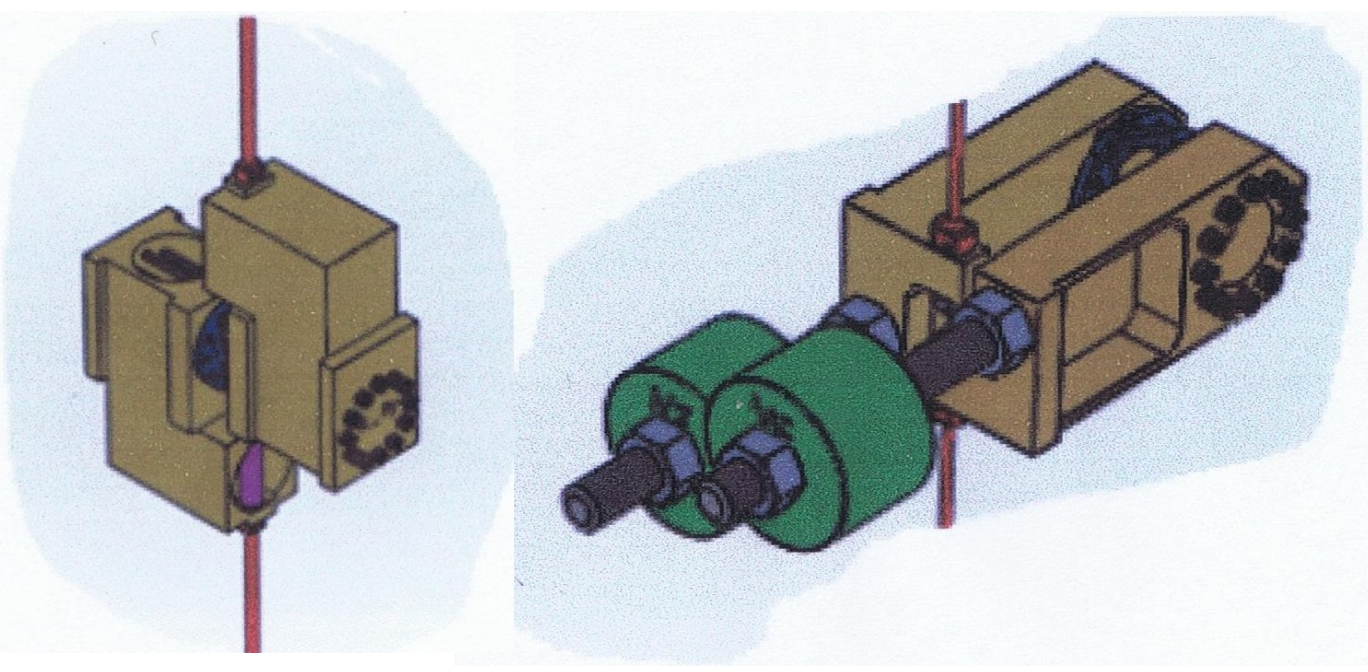

Fig. 3: Mechanical solicitation application adapter for effort Fx and moment Mz.

With these mechanical solicitation application adapter, the sensor is never loaded with all the mechanical solicitation components in the same time. Usually it is loaded with only one elementary mechanical solicitation component or sometimes with 2 elementary mechanical component or 3, but never more. Aside that, metrologicaly, it's not because a mechanical solicitation component is null that this component does not exist. On the contrary, there is roughly more metrological requirements than for a non-null component. Everything has to be done so that the null value of the component be perfectly under control during all the time of the calibration tests. 
The sizes of all the mechanical solicitations application adapters are mainly defined in order that all the identified error causes could not generate relative errors higher than $1 \%$.

Identified error causes taken in account in the sizes calculations are:

- geometrical defects of the tension-compression test machine (misalignment of the effort versus its application line, perpendicular defect of the effort application flange versus the effort application line,

- crosstalk between each mechanical solicitation component and with null effort components (bad control of the null effort components, errors on one of the components due to an other non-null component or to others non-null components if several components are non-null),

- unbalances of the adapters,

- geometrical shape changes during the loading generating misalignments defects or perpendicular defects,

- Length of the levers creating moment's components.

Errors values due to these error causes has been limited to correct values in: calculating large enough dimensions, giving machining quality (surfaces roughness, dimension tolerances) good enough and to manage efficient mechanical decoupling.

Most of errors are reduced in part or in whole in using mechanical decoupling devices. The technic used here is the technique of the use of decoupling rod which apply the efforts to be measured on the sensor. Instead of applying the efforts to be measured directly on the sensor with the gripping tools or the tension-compression test machine effort application flanges, elastic metallic rods with large slenderness are inserted between the test machine clamp and the sensor. Their sizes are calculated so that they generate only small mechanical solicitations lower than the maximum limit allowable when they bear alignment or angular defects at their maximum limits.

With all these cares, uncertainties on measured quantities, especially on reference quantities (reference effort, reference moment) are below the allowed limit.

\subsection{Calibration tests, determination of sensitivity coefficients matrix $\left(b_{i j}\right)$ then of inverse sensitivity coefficients matrix $\left(a_{i j}\right)$}

The sensor is installed on tension-compression test machine successively with each one of the 6 mechanical solicitation elementary components application adapters. Strain gages Wheatstone bridges are connected to an automatic measurement acquisition system controlled by a personal computer like this is shown on the block diagram of figure 4 . 


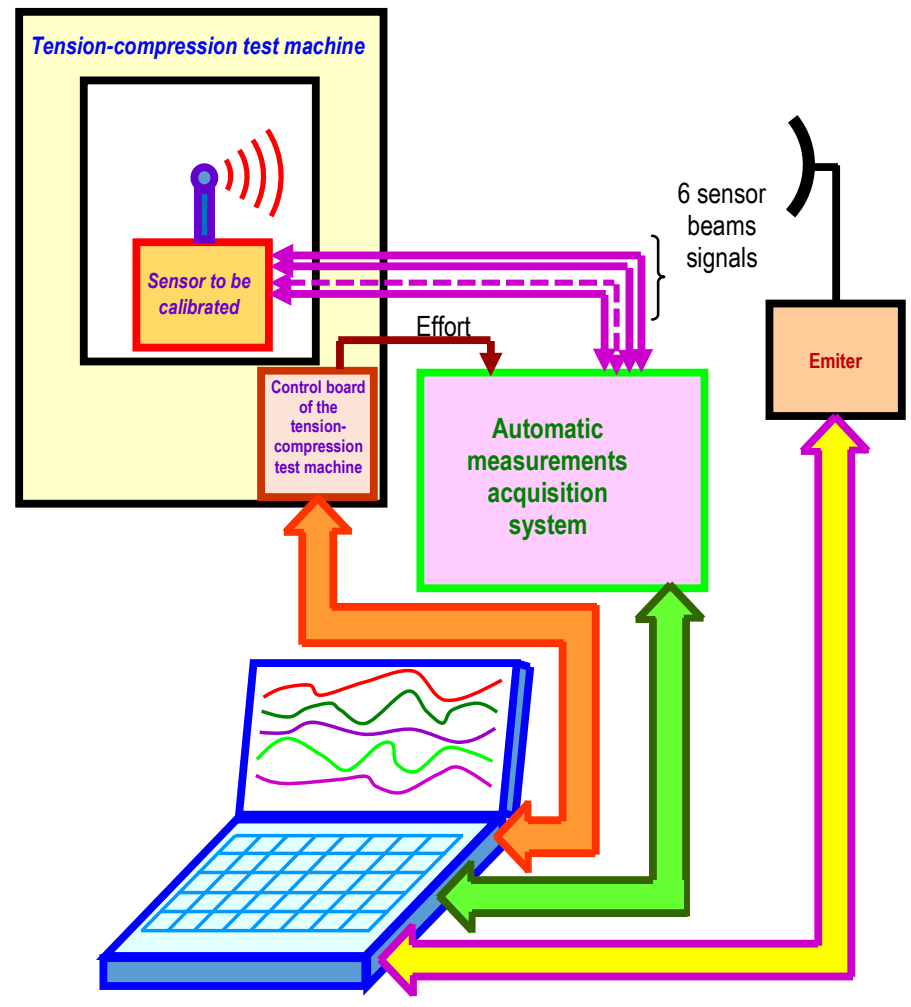

Fig. 4: Block diagram of all the equipment's needed to perform calibration tests.

For each of the 6 calibration tests, tension-compression test machine is also controlled by the personal computer in order to apply loads at various stages in positive (tension) and negative (compression). The dedicated software control the running of the automatic measurements acquisition system (acquisition of tension-compression test machine load cell signal as well as measurements coming from the 6 sensor beams, then, all these measurement values are saved in a file on a computer disk). 


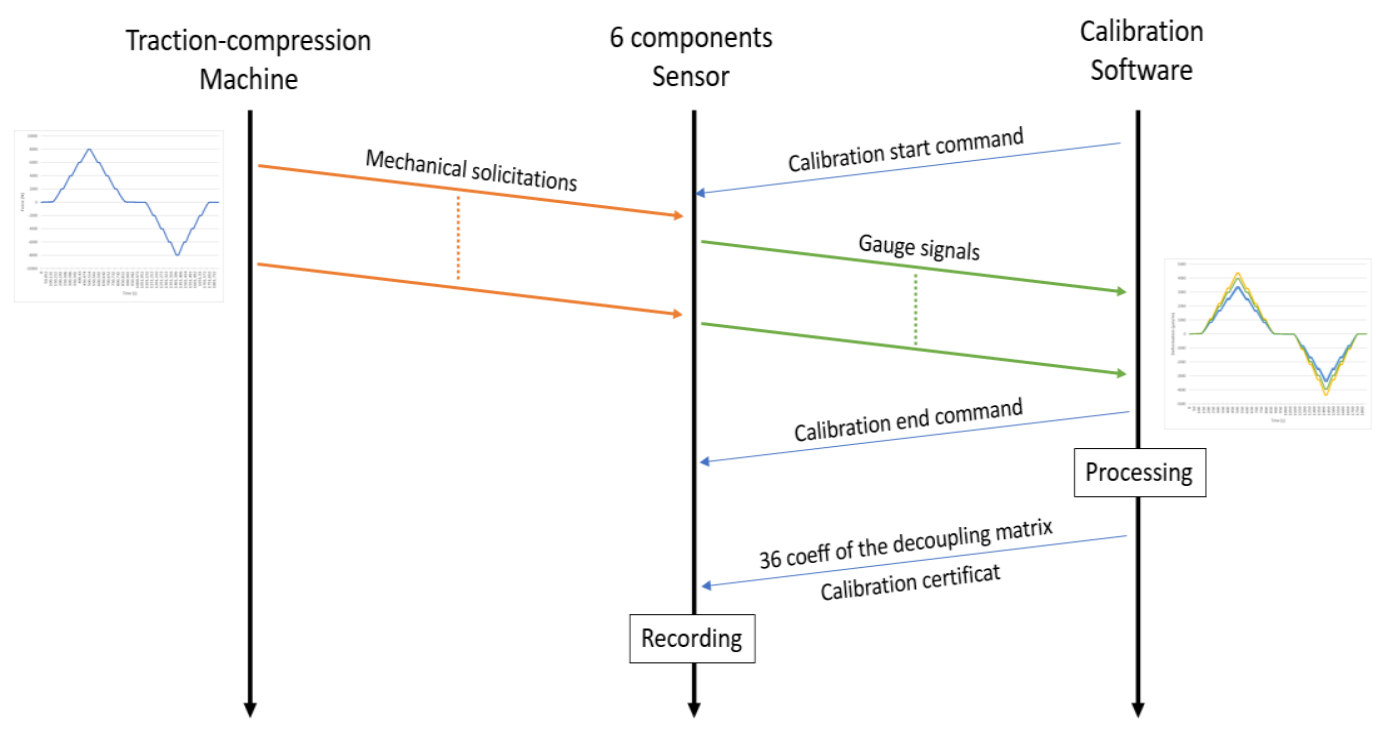

Fig. 5: Functional scheme of calibration processing

Owing to that, a file with one or two columns of reference parameters values (effort and possibly moment) and 6 strain gages Wheatstone bridges signals columns is created at each calibration test.

Strain gages Wheatstone bridges signals evolutions versus reference parameters values are shown on figure 6 . 


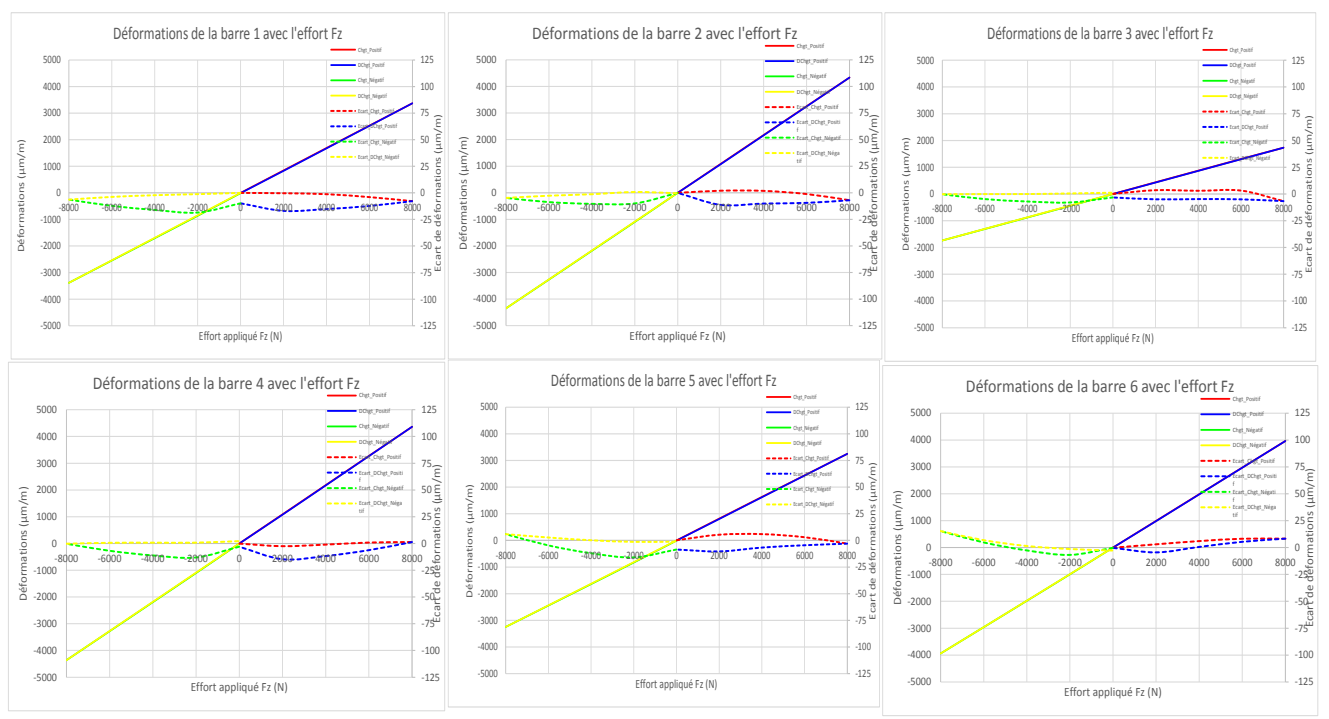

Fig. 6: Strain gages Wheatstone bridges signals evolutions curves got at the end of the calibration test with the elementary mechanical solicitation component Fz.

Each of the 6 sets of curves includes the response curve of a sensor beam (curves having the shape of straight line segments leaning above the abscissa axis with an angle between 20 and $45^{\circ}$ ) and curves of differences between response curves and the minimum square best fit straight lines of these response curves. It appears that the responses of the sensor seem perfectly linear since the curves which represent them are seen, by a human eye, as straight line segments. Difference between response curves and the minimum square best fit straight lines curves show that it's not perfectly true.

There are sets of curves like the ones of figure 6 for each of the 5 others calibration tests. So, there are 36 sets of curves for all.

$\mathrm{b}_{\mathrm{ij}}$ sensitivity coefficients are deduced from these measurement results. So that linear equations system hypothesis which is the basement of this computing method of the sensitivity coefficients, be respected, the calculations are not done directly on measurements results (division of the strain gages Wheatstone bridge values by reference parameters values), but in starting from slopes of the minimum square best fit straight lines.

For some calibration tests (calibration tests with elementary mechanical solicitation component $\mathrm{Fz}$, for instance), computing of sensitivity coefficients is very simple: sensitivity coefficients are directly the values of the slopes of minimum square best fit straight lines.

For other calibration tests, along with the sensor is loaded by more than one elementary mechanical solicitation component, there is several types of response curves slopes which 
are involved in the computation. The calculations are then reduced to the resolution of linear equations systems, most of the time incomplete.

Sensitivity coefficients matrix is got when the measurements results of the 6 calibrations tests have been analyzed.

Invers sensitivity coefficients matrix is then computed in doing the calculations of the matrix inverse of sensitivity coefficients matrix which has just been got in using the improved calculation technique explained in $\S 3$.

All the calculations are performed automatically with the personal computer of figure 4 and a specific software, the block diagram of which being given on figure 7 .

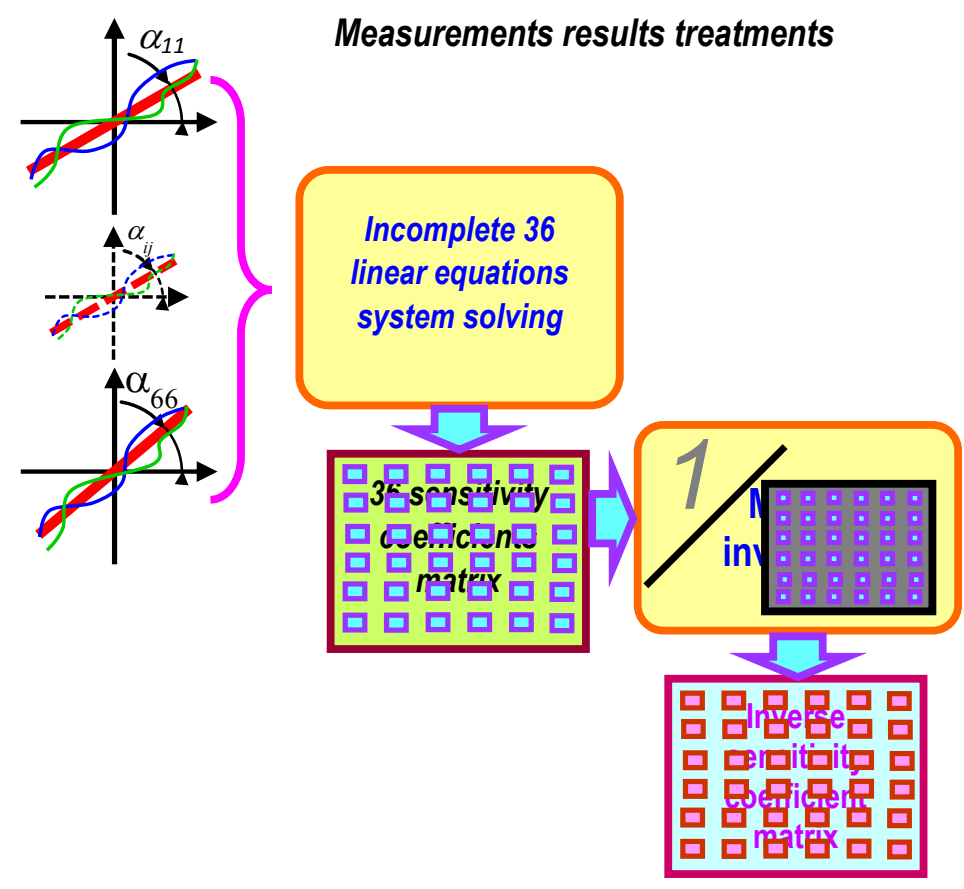

Fig. 7: Sensor components of sensitivity coefficients matrix and inverse sensitivity coefficients matrix calculation block diagram.

After they have been computed, inverse sensitivity coefficients matrix components are downloaded in sensor memory. So that, further, when the sensor is working, it gives directly the 6 components of the mechanical solicitations with which it is loaded (mechanical solicitations to be measured).

\subsection{Calibration tests results retreatment and sensor performance characterization}

With utmost rigor, the calibration of the sensor, strictly according to VIM (International vocabulary of metrology - Basic and general concepts and associated terms) is the comparison of its signals values with the reference values of the mechanical solicitations 
components applied by the tension-compression test machine and mechanical solicitations application adapters during calibration tests.

For that, for each calibration test performed, all the strain gages Wheatstone bridge measurements results are taken again and converted in mechanical solicitations components in using inverse sensibility coefficients matrix. The results of this conversion are compared with the values of the mechanical solicitations components really applied to the sensor all along the calibration tests.

A set of curves representing the differences resulting from these comparisons are drawn for each of the 6 calibration tests.

All these sets of curves are given at figure 8 . 

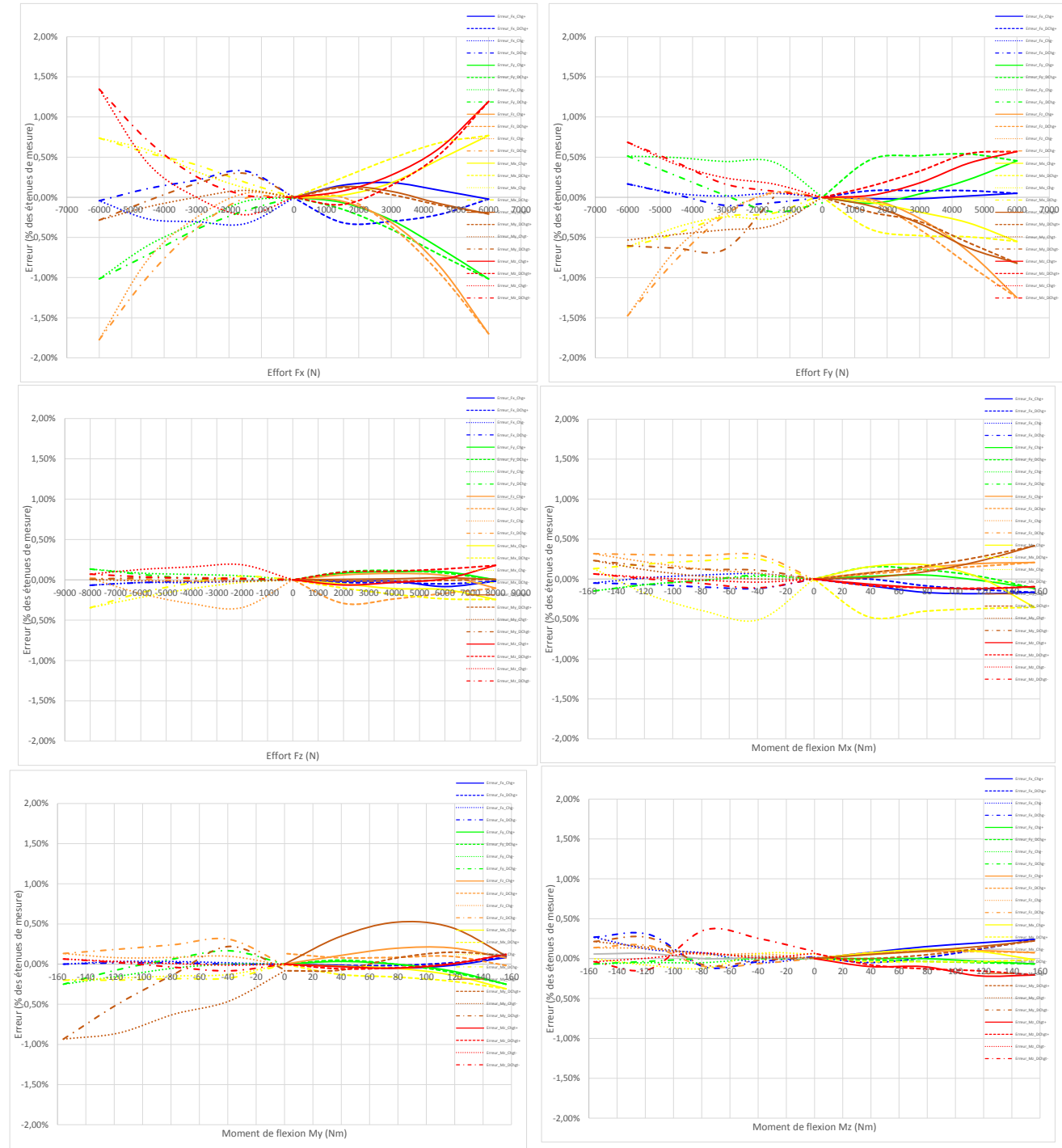

Fig. 8: Difference curves resulting from the comparisons between the values of the mechanical solicitations components given by the sensors and the reference values of the same mechanical solicitation components applied on the sensor by the tension-compression test machine.

Difference values between mechanical solicitations components indicated by the sensor and the ones really applied by tension-compression test machine given on the curves are expressed in relative value relative to the measuring ranges of the sensor. They are expressed in \%.

These curves show that, trueness (non-linearity + hysteresis) on 3 components taken on during calibration tests with components Fx and Fy taken apart, all the maximum differences are below $1 \%$ of the measuring ranges. 
In some calibration tests (calibration tests with $\mathrm{Fz}$ component alone, for instance), the maximum differences are even largely lower. They are less than $0.3 \%$ of the measuring range.

They show also that the differences come mainly from the combination of differences attributed to non-linearity defects and of hysteresis defects.

Differences coming from non-linearity defects may significantly be reduced, or even eliminated, in making corrections, based on the calibration results, even if this is quite complicated in the case of 6 components mechanical solicitations sensors (6 axis effort sensors).

In such case, only the differences coming from hysteresis defects remain. These differences are of $0.3 \%$ of the measuring range at the maximum.

The 3 differences for which the maximum values are higher than $1 \%$ during calibration test with Fx and Fy elementary mechanical solicitations components are: the difference on the $\mathrm{Mz}$ component (which should be of $0 \mathrm{Nm}$ ) the value of which is $1.3 \%$ and the difference on $\mathrm{Fz}$ (which should be of $0 \mathrm{~N}$ too) the maximum value of which is $1.7 \%$, altogether got during the calibration test with the elementary mechanical solicitation component Fx and the difference on the $\mathrm{Fz}$ component, the maximum value of which is $1.5 \%$, got during the calibration test with the elementary mechanical solicitation component Fy.

\section{Comparison with other multi-component forces sensors}

The bibliographical references appearing at the end of this document present several works with multi-component sensors, particularly in the field of biomechanics applied to bicycles. There are many 3-component sensors to measure the forces applied on the pedals. There is also works with 6-component sensors. The presentations focus mainly on measurement results without really giving a clear indication of the uncertainties. In one case, it is also a question of calibration using a device with cables and calibrated masses. The calibration results are not precisely communicated, so that it is not possible to quantify the various defects that seem to exist (non-linearity, interactions between measurement channels, etc.). The authors focused mainly on descriptions of instrumentation, calibration setup and the presentation of test results on bicycles. It appears, however, that the level of uncertainties on the calibration results is clearly greater than $1 \%$ in relative value and sometimes close to $5 \%$. These orders of magnitude are found elsewhere in other articles. The calibration results of the sensor presented here (see Figure 8) show significantly lower errors. Out of 36 error curves, 33 are still less than $1 \%, 27$ are still less than $0.5 \%$ and only 3 are slightly more than $1 \%(1.3,1.5$ and $1.7 \%)$.

\section{Deployment of the sensor to a biomechanical application}

These sensors have been designed for biomechanical applications. Those sensors must have a dynamic response and measurement errors consistent with the study of human movement and performance.

A concrete example of the deployment of these sensors, Figure 9, is the analysis of the high-level cyclist in real-world conditions of practice. To do this, 5 "6-components sensors" will be integrated at the pedals, hanger and saddle. The components of the twisters given by 
these sensors provide input data for algorithms developed to understand, analyze and provide performance indicators of pedaling motion. Information can then be transferred to the cyclist and his coach. Figure 10 illustrates the interaction of these sensors with biomechanical analysis software.

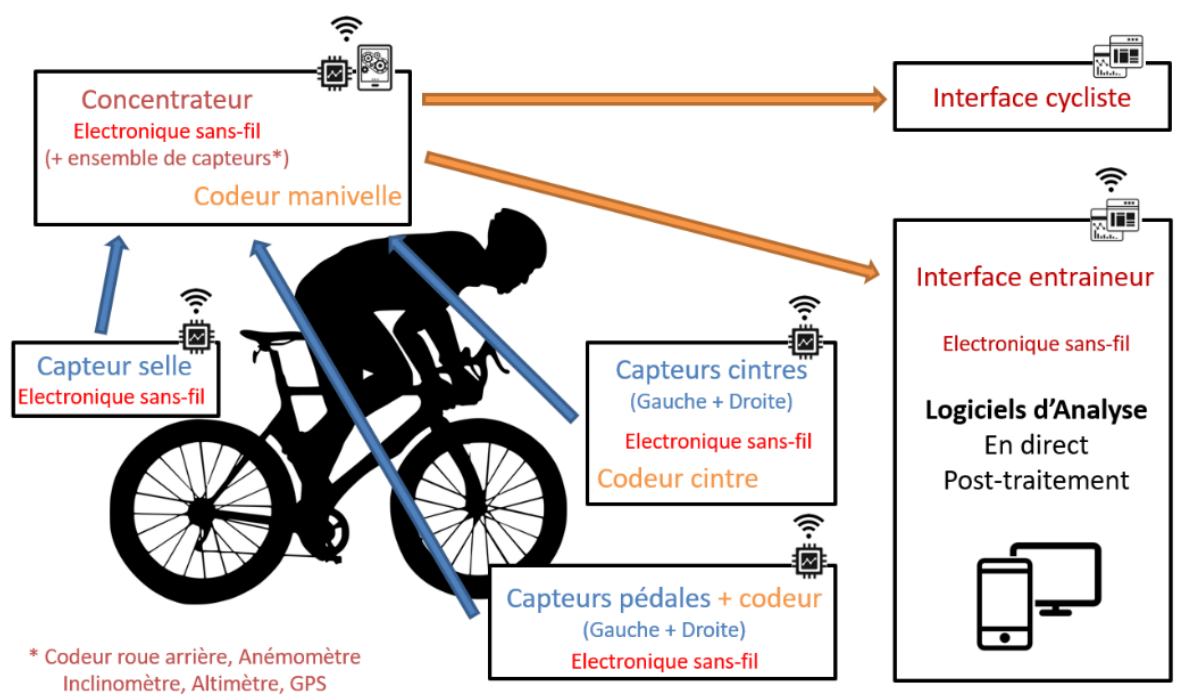

Fig. 9: Functional scheme of transmission chain between sensors and biomechanical software

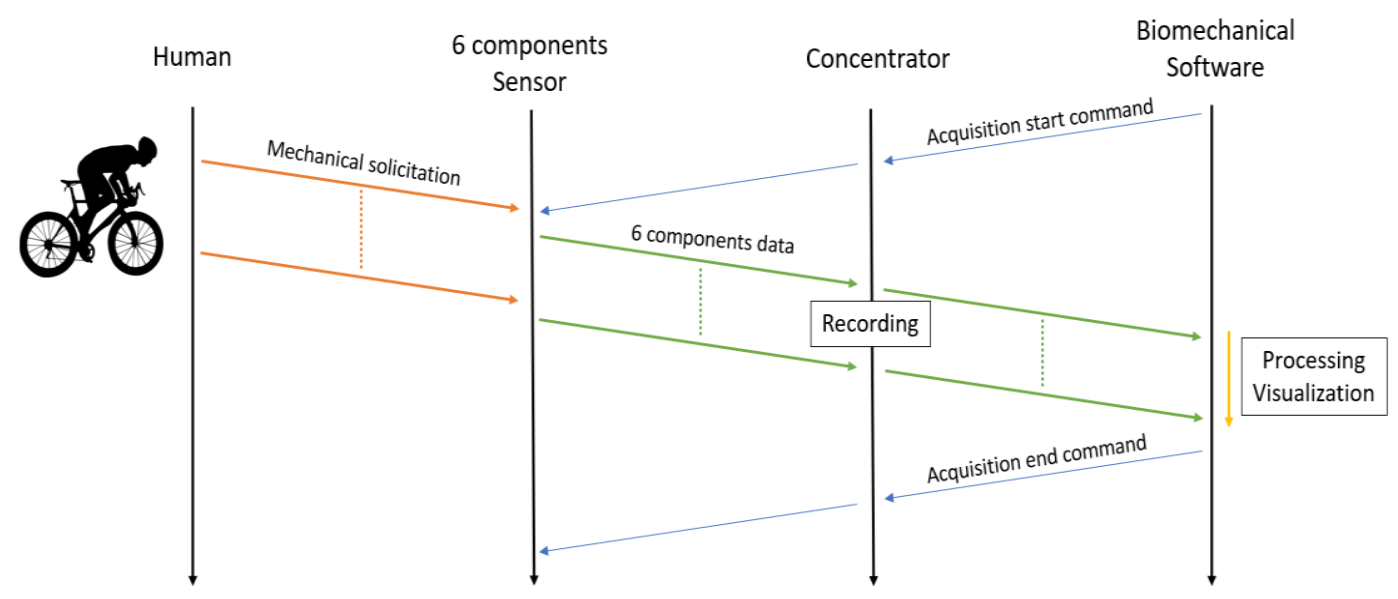

Fig. 10: Functional scheme of biomechanical processing

In practical terms, these sensors can be used on any ergometer intended for sports practice: ergo cycle, ergo kayak, bodybuilding machine, ... 


\section{Prospects uses for industrial applications other than biomechanics}

Already in their current configuration, these sensors have a lot of advantages that predestine them for various industrial uses.

These are sensors easy to manufacture, so inexpensive compared to other types of equivalent sensors, good metrological performance (sensors at $1 \%$ or even $0.5 \%$ ) and, an advantage often overlooked but very important in many industrial uses, high mechanical stiffness as well as state-of-the-art technology, i.e. sensor with an on-board electronics that allows it to have intelligence and to be self-reliant while providing wireless connectivity.

Given these qualities, they can be used in the fields of robotics, machine tool measurement and machining centers, hoisting machine. In the latter case, for example, their "autonomous and wireless connectivity" property can be decisive.

\section{Conclusion}

This project of a 6-component mechanical input sensor based on a 6-beam straight Stewart platform was conducted using the basic concepts of metrology and using only rational techniques; it has enabled the development of a general method of sizing this type of sensor as well as an optimized calibration technique that is efficient and easy to implement.

The sensor thus produced is distinguished from the equivalent sensors by 4 fundamental aspects: it is a sensor with 6 components with effects not mechanically decoupled, it is instrumented by foil resistivestrain gages glued in the middle of straight prismatic bars to get solicitation signals, it has an on-board electronics that provide sensor autonomy. The signals are processed to be converted into the physical quantities and then transmitted wirelessly.

As a result, its cost price is reduced, much lower than the one of equivalent sensors in which the measuring devices of each of the 6 components are completely mechanically decoupled one from each other's. This new sensor is compact and has great mechanical stiffness. Simplified manufacturing is mainly due to the fact that there is no need to decouple measurement signals one from each other's. On the contrary, the sensor is all the more efficient because its measurement signals are the most strongly correlated, but not in any way. Above all, the different laws of correlation should not be identical or similar laws for all measurement signals.

However, these benefits can only be used if it is possible to instantly derive the 6 components of the mechanical solicitations to be measured from the 6 measurement signals provided by the strain gages. Here, this was made possible by the electronics embedded in the sensor.

The 36 beam sensitivity coefficients coming from the calibration tests are downloaded into the on-board sensor's memory.

Thanks to this, the on-board sensor can calculate, at each acquisition, the components of the mechanical solicitationsto be measured. 
This entire process of 6 components effort sensor design, manufacturing and calibration allows to obtain directly sensors whose performance corresponds to the needs of measurements in the field of biomechanics or in the field of current needs of the industry. The first sensors made according to this give measurements results with relative uncertainties in the order of $1 \%$. Improvements of the method of sizing and processing the gross measurement results by correcting the defects of nonlinearity, are possible, which would reduce the uncertainties a little up to about 0.2 to $0.3 \%$.

\section{Bibliographie}

[1], RR. Davis: Measurement of pedal loading in bicycling: I. Instrumentation. J Biomech; 412:843-56 [1981].

[2] ML. Hull, C.Sone Rider/\&bicycle Interaction Loads During Standing Treadmill Cycling. J Biomech; 9:202-218 [1993]

[3] F. Woods, G \& Day, L \& T Withers, R \& H Ilsley, A \& F Maxwell, B. The Dynamic Calibration of Cycle Ergometers. International journal of sports medicine. 15. 168-71. 10.1055/s-2007-1021041 [(1994).].

[4] Gurgel, Jonas \& Porto, Flavia \& Russomano, Thais \& Cambraia, Rodrigo \& Azevedo, Dario \& Glock, F.s \& Carlos Pinheiro Beck, João \& Helegda, Sergio. (2006). Development and Calibration of a Pedal with Force and Moment Sensors. Conference proceedings : ... Annual International Conference of the IEEE Engineering in Medicine and Biology Society. IEEE Engineering in Medicine and Biology Society. Conference. 1. 4144-6. 10.1109/IEMBS.2006.260719.

[5] Stapelfeldt, Björn \& Mornieux, Guillaume \& Oberheim, R \& Belli, Alain \& Gollhofer, Albert. Development and Evaluation of a New Bicycle Instrument for Measurements of Pedal Forces and Power Output in Cycling. International journal of sports medicine. 28. 326-32. 10.1055/s-2006-924352 [2007].

[6] Mimmi, Giovanni \& Rottenbacher, Carlo \& Bonandrini, Giovanni. (2007). Pedalling Strength Analysis in Pathological and Non-pathological Subjects on Cycle-ergometer Instrumented with Three-components Pedals. 18-21.

[7] Vanwalleghem, Joachim \& Mortier, Frederik \& De Baere, Ives \& Loccufier, Mia \& Van Paepegem, Wim. Design of an instrumented bicycle for the evaluation of bicycle dynamics and its relation with the cyclist's comfort. Procedia Engineering. 34. 485-490. 10.1016/j.proeng.2012.04.083 [2012].

[8] Balbinot, A \& Milani, Cleiton \& Nascimento, Jussan. A New Crank Arm-Based Load Cell for the 3D Analysis of the Force Applied by a Cyclist. Sensors (Basel, Switzerland). 14. 22921-22939. 10.3390/s141222921 [2014].

[9] V. Omar, D. Rafael, C. Vinicius and B. Alexandre, "Complete factorial design experiment for 3D load cell instrumented crank validation," 37th Annual International Conference of the IEEE Engineering in Medicine and Biology Society (EMBC), Milan, 2015, pp. 3655-3658. 10.1109/EMBC.2015.7319185 [2015]. 
[10] I. Kraemer, Luke \& D. Gutierrez-Franco, Juan \& E. Deschamps, Jake \& C. Dudum, Karim \& Dandekar, Eshan \& Hazelwood, Scott \& V. Porumamilla, Hemanth \& M. Klisch, Stephen. Design and implementation of an instrumented pedal for cycling biomechanics research [2015].

[11] Bibbo, Daniele \& Gabriele, Stefano \& Scorza, Andrea \& Schmid, Maurizio \& Sciuto, Salvatore \& Conforto, Silvia. Strain gauges position optimization in designing custom load cells for sport gesture analysis. 1-6. 10.1109/HealthCom.2018.8531106 [2018].

[12] Bibbo, Daniele \& Gabriele, Stefano \& Scorza, Andrea \& Schmid, Maurizio \& Sciuto, Salvatore \& Conforto, Silvia. A Novel Technique to Design and Optimize Performances of Custom Load Cells for Sport Gesture Analysis. IRBM. 10.1016/j.irbm.2019.05.005 [2019]. 